\title{
NEW FAULT TOLERANT CONTROL STRATEGIES FOR NONLINEAR TAKAGI-SUGENO SYSTEMS
}

\author{
DALIL ICHALAL * ${ }^{*}$ Benoît MARX ${ }^{* *}$, José RAGOT $^{* *}$, DIDIER MAQUIN ** \\ * IBISC Laboratory \\ University of Evry Val d'Essonne, 40, rue du Pelvoux, 91020 Courcouronnes, France \\ e-mail: dalil.ichalal@ibisc.univ-evry.fr \\ ${ }^{* *}$ Research Centre for Automatic Control of Nancy (CRAN) \\ University of Lorraine, 2, avenue de la forêt de Haye, 54516 Vandoeuvre-les-Nancy, France \\ e-mail: \{benoit.marx, jose.ragot, didier.maquin\}@univ-lorraine.fr
}

\begin{abstract}
New methodologies for Fault Tolerant Control (FTC) are proposed in order to compensate actuator faults in nonlinear systems. These approaches are based on the representation of the nonlinear system by a Takagi-Sugeno model. Two control laws are proposed requiring simultaneous estimation of the system states and of the occurring actuator faults. The first approach concerns the stabilization problem in the presence of actuator faults. In the second, the system state is forced to track a reference trajectory even in faulty situation. The control performance depends on the estimation quality; indeed, it is important to accurately and rapidly estimate the states and the faults. This task is then performed with an Adaptive Fast State and Fault Observer (AFSFO) for the first case, and a Proportional-Integral Observer (PIO) in the second. Stability conditions are established with Lyapunov theory and expressed in a Linear Matrix Inequality (LMI) formulation to ease the design of FTC. Furthermore, relaxed stability conditions are given with the use of Polya's theorem. Some simulation examples are given in order to illustrate the proposed approaches.
\end{abstract}

Keywords: Takagi-Sugeno model, fault tolerant control, simultaneous fault and state estimations, Polya's theorem, Lyapunov theory, input-to-state stability.

\section{Introduction}

For several years, the problem of fault tolerance has been treated from many points of view. Two classes can be considered: passive control and active control. The formed may be viewed as robust control. It requires a priori knowledge of possible faults which may affect the system. The principal idea of this kind of control is based on treating all possible faults as uncertainties which are taken into account for the design of tolerant control by using different techniques such as $\mathcal{H}_{\infty}$ (Patton, 1997; Niemann and Stoustrup, 2005). The interest of this approach lies in the fact that no online information is needed and the structure of the control law remains unchanged. Generally, the structure of the uncertainties (faults) is not taken into account in order to lead to a convex optimization problem. Furthermore, the class of the faults considered is limited and it then becomes risky to use only passive fault tolerant control (see Mufeed et al., 2003).

The second class concerns active fault tolerant con- trol, which is more interesting due to its possibility to take into account a large class of faults, because of its variable structure, which may change in the presence of faults. The knowledge of some information about these is required and obtained from a Fault Detection and Diagnosis (FDD) block. Different ideas are developed in the literature, e.g., control law re-scheduling (OcampoMartinez et al., 2010; Leith and Leithead, 1999; Stilwell and Rugh, 1997). This approach requires a very robust Fault Detection and Isolation (FDI) block, which constitutes its major disadvantage. Indeed, a false alarm or an undetected fault may lead to degraded performance or even to instability. Other smooth fault tolerant control laws are proposed by Ichalal et al. (2010) for TakagiSugeno systems and by Patton and Klinkhieo (2009a) for LPV systems.

Many efforts have been spent on the problem of designing active fault tolerant controllers for nonlinear systems, and the obtained results are linked to the system rep- 
resentation. Some works can be mentioned in the FTC field for nonlinear systems. For example, Gao and Ding (2007) took into account actuator faults for nonlinear descriptor systems with Lipschitz nonlinearities. In the work of Ocampo-Martinez et al. (2010), a method which requires only fault isolation was proposed for T-S systems. It was formed by a bank of observer based controllers. A switching mechanism is then designed depending on the obtained residuals. An efficient way to deal with system nonlinearity in FTC design is the T-S approach introduced by Takagi and Sugeno (1985). This approach is used by Ichalal et al. (2010), who employ FTC based on trajectory tracking and Proportional-Integral Observer (PIO) design for T-S systems with weighting functions depending on the state of the system which is not accessible for measurements.

In this paper, a new actuator fault tolerant control technique is proposed. Using a fast adaptive observer proposed by Zhang et al. (2008) and extended here to nonlinear T-S systems, the state and the fault affecting the system are rapidly estimated. The use of such an observer is motivated by the fact that, if a fault occurs, it is important to quickly and accurately detect it in order to take it into account and preserve the system performances. With the use of Lyapunov theory, sufficient conditions are obtained for asymptotic stability in the constant fault case and for Input-to-State Stability (ISS) in the case of time varying faults. The LMI formulation is used for representing the obtained stability conditions in an adequate form for existing LMI solvers. Relaxed stability conditions are obtained with the use of Polya's theorem (Sala and Ariño, 2007). Finally, another FTC strategy is proposed, based on trajectory tracking. Put in other words, the system state is kept as close as possible to a reference state, even in a faulty situation. Some simulations illustrate the obtained results.

\section{Takagi-Sugeno structure for modeling}

T-S modeling allows representing the behavior of nonlinear systems by the interpolation of a set of linear submodels. Each sub-model contributes to the global behavior of the nonlinear system through a weighting function $\mu_{i}(\xi(t))$. The T-S structure is given by

$$
\left\{\begin{array}{l}
\dot{x}(t)=\sum_{i=1}^{r} \mu_{i}(\xi(t))\left(A_{i} x(t)+B_{i} u(t)\right), \\
y(t)=C x(t)
\end{array}\right.
$$

where $x(t) \in \mathbb{R}^{n}$ is the state vector, $u(t) \in \mathbb{R}^{n_{u}}$ is the input vector, $y(t) \in \mathbb{R}^{n_{y}}$ represents the output vector. $A_{i} \in \mathbb{R}^{n \times n}, B_{i} \in \mathbb{R}^{n \times n_{u}}$ and $C \in \mathbb{R}^{n_{y} \times n}$ are known matrices. The functions $\mu_{i}(\xi(t))$ are the weighting functions depending on the variable $\xi(t)$, which is, in the sequel of the paper, accessible for measurements (as the input or the output of the system). These functions satisfy the following properties:

$$
\left\{\begin{array}{l}
\sum_{i=1}^{r} \mu_{i}(\xi(t))=1, \\
0 \leq \mu_{i}(\xi(t)) \leq 1, \quad \forall i \in\{1,2, \ldots, r\} .
\end{array}\right.
$$

Obtaining a T-S model (1) can be performed with various methods such as linearization around some operating points and using adequate weighting functions. It can also be obtained by black-box approaches, which allow identifying the parameters of the model from inputoutput data. Finally, a T-S model can be obtained from the well-known nonlinear sector transformations (Tanaka and Wang, 2001; Nagy et al., 2009). These transformations allow obtaining an exact $\mathrm{T}-\mathrm{S}$ representation of a nonlinear model with no information loss on a compact set of the state space.

Thanks to the convex sum property of the weighing functions (2), it is possible to generalize some tools developed in the linear domain to nonlinear systems. The representation (1) is very interesting in the sense that it simplifies the stability studies of nonlinear systems and the design of control laws and observers. In the works of Tanaka and Wang (2001) as well as Kruszewski et al. (2008), the stability and stabilization tools are inspired from the study of linear systems. Akhenak et al. (2008) and Marx et al. (2007), worked on the problem of state estimation and diagnosis of T-S systems. The approaches proposed in these last mentioned papers rely on the generalization of the classical observers (Luenberger observer) (Luenberger, 1971) and Unknown Input Observer (UIO) (Darouach et al., 1994)) to nonlinear systems. Sala and Ariño (2007) proposed a new approach, derived from Polya's theorem, which leads to asymptotic necessary and sufficient stability conditions. FTC was also envisaged for T-S systems with time-delay by Zhang et al. (2009), but no reference tracking was considered.

In the remainder of the paper, the following two lemmas and notations are used.

Lemma 1. Consider two matrices $X$ and $Y$ with appropriate dimensions and $G$ as a symmetric positive definite matrix. The following property is satisfied:

$$
X^{T} Y+Y^{T} X \leq X^{T} G X+Y^{T} G^{-1} Y, \quad G>0 .
$$

Lemma 2. (Congruence) Consider two matrices $P$ and $Q$. If $P$ is positive definite and if $Q$ is a full column rank matrix, then the matrix $Q P Q^{T}$ is positive definite.

Notation. For any square matrix $M, \lambda_{\max }(M)$ represents the maximum singular value of the matrix $M$ and $\mathbb{S}(M)$ is defined by $\mathbb{S}(M)=M+M^{T}$. A block diagonal matrix with the block matrices $M_{i}$ on the diagonal entries is denoted by $\operatorname{diag}\left(M_{1}, M_{2}, \ldots, M_{n}\right)$. In a partitioned matrix, the $\operatorname{star}^{\prime} *$ ' denotes the terms induced by symmetry. 


\section{Problem statement}

Under actuator faults, the system (1) can be rewritten in the following form:

$$
\left\{\begin{array}{l}
\dot{x}(t)=\sum_{i=1}^{r} \mu_{i}(\xi(t))\left(A_{i} x(t)+B_{i}(u(t)+f(t))\right), \\
y(t)=C x(t)
\end{array}\right.
$$

where $f(t)$ is an actuator fault. Faults can affect a system in many different ways. They can be represented by an additive or a multiplicative external signal. It can be pointed out that, if the fault depends on the system state, it can change the model structure and cause its instability. For instance, malfunctions of an actuator can be represented by a faulty control input defined by $u_{f}(t)=$ $\left(I_{n_{u}}-\gamma\right) u(t)$, which can easily be rewritten as an external additive signal $(u(t)+f(t))$ with $f(t)=-\gamma u(t)$ and $\gamma=\operatorname{diag}\left(\gamma_{1}, \gamma_{2}, \ldots, \gamma_{n_{u}}\right), 0 \leq \gamma_{i} \leq 1\left(i=1, \ldots, n_{u}\right)$, where

$\left\{\begin{array}{l}\gamma_{i}=1 \Rightarrow \text { a total failure of the } i \text {-th actuator, } \\ \gamma_{i}=0 \Rightarrow \text { the } i \text {-th actuator is healthy, } \\ \left.\gamma_{i} \in\right] 01[\Rightarrow \text { a loss of effectiveness of the } i \text {-th actuator. }\end{array}\right.$

For example, if $\gamma_{2}=0.4$, there is a $40 \%$ loss of effectiveness of the second actuator. Note that such multiplicative faults can cause system instability.

Assumption 1. In this paper, it is assumed that

A1. The faults have a norm bounded first time derivative

$$
\|\dot{f}(t)\| \leq f_{1 \max }, \quad 0 \leq f_{1 \max }<\infty .
$$

A2. $\operatorname{rank}\left(C B_{i}\right)=n_{u}, i=1, \ldots, r$.

A3. Only partial actuator failures are considered, i.e., $\gamma_{i} \in[01[, i=1, \ldots, r$.

The objective of FTC design is to find a control law $u(t)$ in (4) such that the system remains stable even in a faulty case. For this purpose, state and fault estimates will be used in order to minimize the fault influence on system stability.

\section{Stabilizing fault tolerant control for nonlinear systems}

In order to estimate the state and the faults of the system (4), the following adaptive observer is proposed:

$$
\left\{\begin{aligned}
\dot{\hat{x}}(t)= & \sum_{i=1}^{r} \mu_{i}(\xi(t))\left(A_{i} \hat{x}(t)+B_{i}(u(t)+\hat{f}(t))\right. \\
& \left.+L_{i} e_{y}(t)\right), \\
\hat{y}(t)= & C \hat{x}(t), \\
\dot{\hat{f}}(t)= & \Gamma \sum_{i=1}^{r} \mu_{i}(\xi(t)) F_{i}\left(\dot{e}_{y}(t)+\sigma e_{y}(t)\right), \\
e_{y}(t)= & y(t)-\hat{y}(t),
\end{aligned}\right.
$$

and the active fault tolerant control is chosen as

$$
u(t)=-\sum_{i=1}^{r} \mu_{i}(\xi(t)) K_{i} \hat{x}(t)-\hat{f}(t) .
$$

This control law can be seen as a generalization to the nonlinear case of the approach proposed by Patton and Klinkhieo (2009b). The solution of the FTC problem is obtained by setting $\Gamma \in \mathbb{R}^{n_{u} \times n_{u}}$ and the scalars $\sigma$ and $\beta \in \mathbb{R}$, and determining the gains $L_{i} \in \mathbb{R}^{n \times n_{y}}, F_{i} \in$ $\mathbb{R}^{n_{u} \times n_{y}}$ and $K_{i} \in \mathbb{R}^{n_{u} \times n}$ by LMI optimization such that the state of the system asymptotically converges to zero if the fault $f(t)$ is constant or to a small set around the origin when $f(t)$ is time varying with a norm bounded first time derivative. The expression describing the dynamics of the estimated fault $\hat{f}(t)$ given in (6) depends on both the output error and its derivative.

Let us consider the state and fault estimation errors defined respectively by

$$
\begin{aligned}
& e_{x}(t)=x(t)-\hat{x}(t), \\
& e_{f}(t)=f(t)-\hat{f}(t) .
\end{aligned}
$$

The dynamics of the state estimation error and the closedloop system with the control (7) obey the differential equations:

$$
\begin{aligned}
\dot{e}_{x}(t)= & \sum_{i=1}^{r} \mu_{i}(\xi(t))\left(\Phi_{i} e_{x}(t)+B_{i} e_{f}(t)\right), \\
\dot{x}(t)= & \sum_{i=1}^{r} \sum_{j=1}^{r} \mu_{i}(\xi(t)) \mu_{j}(\xi(t))\left(\Xi_{i j} x(t)\right. \\
& \left.+B_{i} e_{f}(t)+B_{i} K_{j} e_{x}(t)\right),
\end{aligned}
$$

where $\Phi_{i}=A_{i}-L_{i} C$ and $\Xi_{i j}=A_{i}-B_{i} K_{j}$.

Theorem 1. Under Assumption 1, given positive scalars $\sigma$ and $\beta$, if there exists symmetric and positive definite matrices $\mathcal{X} \in \mathbb{R}^{n \times n}, P_{2} \in \mathbb{R}^{n \times n}$, a positive definite matrix $G \in \mathbb{R}^{n_{u} \times n_{u}}$, matrices $M_{i} \in \mathbb{R}^{n_{u} \times n}$ and $N_{i} \in \mathbb{R}^{n \times n_{y}}$ and a positive scalar $\eta$ solution to the optimization problem

$$
\min \eta
$$

subject to

$$
\begin{gathered}
\left(\begin{array}{ccccc}
\eta I & B_{i}^{T} P_{2}-F_{i} C \\
* & & \eta
\end{array}\right)>0 \\
\mathcal{Q}_{i j}=\left(\begin{array}{ccccc}
S_{i j} & B_{i} M_{j} & B_{i} & 0 & 0 \\
* & -2 \beta \mathcal{X} & 0 & \beta I & 0 \\
* & * & -2 \beta I & 0 & \beta I \\
* & * & * & \Omega_{i} & \mathcal{R}_{i j} \\
* & * & * & * & \Psi_{i j}
\end{array}\right)<0,
\end{gathered}
$$




$$
\begin{aligned}
S_{i j} & =\mathbb{S}\left(A_{i} \mathcal{X}-B_{i} M_{j}\right), \\
\Omega_{i} & =\mathbb{S}\left(P_{2} A_{i}-N_{i} C\right), \\
\mathcal{R}_{i j} & =-\frac{1}{\sigma}\left(A_{j}^{T} P_{2}-C^{T} N_{j}^{T}\right) B_{i}, \\
\Psi_{i j} & =-\frac{1}{\sigma}\left(B_{i}^{T} P_{2} B_{j}+B_{j}^{T} P_{2} B_{i}\right)+\frac{1}{\sigma} G,
\end{aligned}
$$

then the state $x(t)$ of the system, the state estimation error $e_{x}(t)$ and the fault estimation error $e_{f}(t)$ are bounded. Furthermore, if the bound of the first time derivative of $f(t)$ is zero, i.e., $f_{1 \max }=0$, these variables converge asymptotically to zero. The gains of the observer and fault tolerant control are given by $F_{i}, L_{i}=P_{2}^{-1} N_{i}$ and $K_{i}=$ $M_{i} \mathcal{X}^{-1}$.

Proof. In order to prove both the stability of the closedloop system and the convergence of the state and fault estimation errors, consider a Lyapunov function depending on $x(t), e_{x}(t)$ and $e_{f}(t)$ defined by

$$
\begin{aligned}
V(t)= & x^{T}(t) P_{1} x(t)+e_{x}^{T}(t) P_{2} e_{x}(t) \\
& +\frac{1}{\sigma} e_{f}(t) \Gamma^{-1} e_{f}(t),
\end{aligned}
$$

where $P_{1}, P_{2}$ and $\Gamma$ are symmetric and positive definite matrices with appropriate dimensions.

According to Eqns. (10) and (11), the time derivative of $V(t)$ is given by

$$
\begin{aligned}
\dot{V}(t)= & \sum_{i=1}^{r} \sum_{j=1}^{r} \mu_{i}(\xi(t)) \mu_{j}(\xi(t))\left(x^{T}(t) \Pi_{i j} x(t)\right. \\
& +e_{x}^{T}(t) \Omega_{i} e_{x}(t)+2 x^{T}(t) P_{1} B_{i} K_{j} e_{x}(t) \\
& +2 x^{T}(t) P_{1} B_{i} e_{f}(t)+2 e_{x}^{T}(t) P_{2} B_{i} e_{f}(t) \\
& \left.+\frac{2}{\sigma} e_{f}^{T}(t) \Gamma^{-1} \dot{e}_{f}(t)\right)
\end{aligned}
$$

where

$$
\begin{aligned}
\Pi_{i j} & =\Xi_{i j}^{T} P_{1}+P_{1} \Xi_{i j}, \\
\Omega_{i} & =\Phi_{i}^{T} P_{2}+P_{2} \Phi_{i} .
\end{aligned}
$$

Knowing that $\dot{e}_{f}(t)=\dot{f}(t)-\dot{\hat{f}}(t)$ and using the expression of $\hat{f}(t)$ in (6), we obtain

$$
\begin{aligned}
\dot{V}(t)= & \sum_{i=1}^{r} \sum_{j=1}^{r} \mu_{i}(\xi(t)) \mu_{j}(\xi(t))\left(x^{T}(t) \Pi_{i j} x(t)\right. \\
& +e_{x}^{T}(t) \Omega_{i} e_{x}(t)+2 x^{T}(t) P_{1} B_{i} K_{j} e_{x}(t) \\
& +2 x^{T}(t) P_{1} B_{i} e_{f}(t)+2 e_{x}^{T}(t) P_{2} B_{i} e_{f}(t) \\
& -\frac{2}{\sigma} e_{f}^{T}(t) F_{i}\left(\dot{e}_{y}(t)+\sigma e_{y}(t)\right) \\
& \left.+\frac{2}{\sigma} e_{f}^{T}(t) \Gamma^{-1} \dot{f}(t)\right)
\end{aligned}
$$

where $e_{y}(t)=C e_{x}(t)$.
Using the differential equation (10) generating $e_{x}(t)$, the following is obtained:

$$
\begin{aligned}
\dot{V}(t)= & \sum_{i=1}^{r} \sum_{j=1}^{r} \mu_{i}(\xi(t)) \mu_{j}(\xi(t))\left(x^{T}(t) \Pi_{i j} x(t)\right. \\
& +e_{x}^{T}(t) \Omega_{i} e_{x}(t)+2 x^{T}(t) P_{1} B_{i} K_{j} e_{x}(t) \\
& +2 x^{T}(t) P_{1} B_{i} e_{f}(t)+2 e_{x}^{T}(t) P_{2} B_{i} e_{f}(t) \\
& -\frac{2}{\sigma} e_{f}^{T}(t) F_{i} C \Phi_{j} e_{x}(t)-\frac{2}{\sigma} e_{f}^{T}(t) F_{i} C B_{j} e_{f}(t) \\
& \left.-2 e_{f}^{T}(t) F_{i} C e_{x}(t)+\frac{2}{\sigma} e_{f}^{T}(t) \Gamma^{-1} \dot{f}(t)\right) .
\end{aligned}
$$

Using Lemma 1 and Assumption A1, we deduce that

$$
\begin{aligned}
& 2 \frac{1}{\sigma} e_{f}^{T}(t) \Gamma^{-1} \dot{f}(t) \\
& \leq \frac{1}{\sigma} e_{f}^{T}(t) G e_{f}+\frac{1}{\sigma} \dot{f}^{T}(t) \Gamma^{-1} G^{-1} \Gamma^{-1} \dot{f}(t) \\
& \leq \frac{1}{\sigma} e_{f}^{T}(t) G e_{f}+\frac{1}{\sigma} f_{1 \max }^{2} \lambda_{\max }\left(\Gamma^{-1} G^{-1} \Gamma,^{-1}\right)
\end{aligned}
$$

and using Assumption A2, it is possible to obtain $F_{i}$ and $P_{2}$ such that $B_{i}^{T} P_{2}=F_{i} C$. The time derivative of the Lyapunov function (24) is bounded as follows:

$$
\dot{V}(t) \leq \tilde{x}^{T}(t) \sum_{i=1}^{r} \sum_{j=1}^{r} \mu_{i}(\xi(t)) \mu_{j}(\xi(t)) \Delta_{i j} \tilde{x}(t)+\delta,
$$

where

$$
\begin{gathered}
\tilde{x}^{T}(t)=\left(x^{T}(t) e_{x}^{T}(t) e_{f}^{T}(t)\right)^{T}, \\
\delta=\frac{1}{\sigma} f_{1 \max }^{2} \lambda_{\max }\left(\Gamma^{-1} G^{-1} \Gamma^{-1}\right), \\
\Delta_{i j}=\left(\begin{array}{ccc}
\Pi_{i j} & P_{1} B_{i} K_{j} & P_{1} B_{i} \\
* & \Omega_{i} & -\frac{1}{\sigma} \Phi_{j}^{T} P_{2} B_{i} \\
* & * & \Psi_{i j}
\end{array}\right) .
\end{gathered}
$$

If the following inequality holds:

$$
\sum_{i=1}^{r} \sum_{j=1}^{r} \mu_{i}(\xi(t)) \mu_{j}(\xi(t)) \Delta_{i j}<0,
$$

it is established that

$$
\dot{V}(t)<-\varepsilon\|\tilde{x}(t)\|^{2}+\delta,
$$

where $\varepsilon>0$ is defined by

$$
\varepsilon=\min _{t>0} \lambda_{\min }\left(-\sum_{i=1}^{r} \sum_{j=1}^{r} \mu_{i}(\xi(t)) \mu_{j}(\xi(t)) \Delta_{i j}\right),
$$

which can also be bounded by

$$
\varepsilon \leq \min _{i, j} \lambda_{\min }\left(-\Delta_{i j}\right)
$$


It follows that $\dot{V}(t)<0$ if $\varepsilon\|\tilde{x}(t)\|^{2}>\delta$, and according to Lyapunov stability theory the state $x(t)$, the state estimation error $e_{x}(t)$ and the fault estimation error $e_{f}(t)$ converge to a small set around the origin and then lie in it. This set is smaller as the constant $\delta$ converges to zero.

In order to complete the proof, it remains to establish some LMI conditions to ensure that (30) and $B_{i}^{T} P_{2}=$ $F_{i} C$ hold. The latter is first considered.

As pointed out by Zhang et al. (2008), it is difficult to solve simultaneously the inequality

$$
\sum_{i=1}^{r} \sum_{j=1}^{r} \mu_{i}(\xi(t)) \mu_{j}(\xi(t)) \Delta_{i j}<0
$$

with the equality constraint $B_{i}^{T} P_{2}=F_{i} C$. A technique for reducing this difficulty is to formulate the equality constraint as an optimization problem (Corless and Tu, 1998):

$$
\min \eta
$$

subject to

$$
\left(\begin{array}{cc}
\eta I & B_{i}^{T} P_{2}-F_{i} C \\
* & \eta I
\end{array}\right)>0
$$

For simplicity, the following notation will be used:

$$
\begin{aligned}
Y_{\xi} & =\sum_{i=1}^{r} \mu_{i}(\xi(t)) Y_{i}, \\
Y_{\xi \xi} & =\sum_{i=1}^{r} \sum_{i=1}^{r} \mu_{i}(\xi(t)) \mu_{j}(\xi(t)) Y_{i j},
\end{aligned}
$$

where $Y_{i}$ and $Y_{i j}$ are given matrices. Using this representation, the inequality (30) becomes

$$
\Delta_{\xi \xi}=\left(\begin{array}{cc}
\Pi_{\xi \xi} & \Theta_{\xi \xi} \\
\Theta_{\xi \xi}^{T} & \Lambda_{\xi \xi}
\end{array}\right)<0
$$

where

$$
\begin{gathered}
\Theta_{i j}=\left(\begin{array}{cc}
P_{1} B_{i} K_{j} & P_{1} B_{i}
\end{array}\right), \\
\Lambda_{i j}=\left(\begin{array}{cc}
\Omega_{i} & -\frac{1}{\sigma} \Phi_{j}^{T} P_{2} B_{i} \\
* & \Psi_{i j}
\end{array}\right) .
\end{gathered}
$$

Consider a symmetric matrix $X$ defined as follows:

$$
X=\left(\begin{array}{cc}
P_{1}^{-1} & 0 \\
0 & X_{1}
\end{array}\right), \quad X_{1}=\left(\begin{array}{cc}
P_{1}^{-1} & 0 \\
0 & I
\end{array}\right) \text {. }
$$

Using Lemma 2 , post and pre-multiplying the inequality (37) by $X$, it follows that (37) is equivalent to the following inequality:

$$
\left(\begin{array}{cc}
P_{1}^{-1} \Pi_{\xi \xi} P_{1}^{-1} & P_{1}^{-1} \Theta_{\xi \xi} X_{1} \\
* & X_{1} \Lambda_{\xi \xi} X_{1}
\end{array}\right)<0 .
$$

Since the following inequality holds for any scalar $\beta$ :

$$
\begin{gathered}
\left(X_{1}+\beta \Lambda_{\xi \xi}^{-1}\right)^{T} \Lambda_{\xi \xi}\left(X_{1}+\beta \Lambda_{\xi \xi}^{-1}\right) \leq 0 \\
\Leftrightarrow X_{1} \Lambda_{\xi \xi} X_{1} \leq-2 \beta X_{1}-\beta^{2} \Lambda_{\xi \xi}^{-1},
\end{gathered}
$$

and with a Schur complement, it follows that the inequality (41) holds if

$$
\left(\begin{array}{ccc}
P_{1}^{-1} \Pi_{\xi \xi} P_{1}^{-1} & P_{1}^{-1} \Theta_{\xi \xi} X_{1} & 0 \\
* & -2 \beta X_{1} & \beta I \\
* & * & \Lambda_{\xi \xi}
\end{array}\right)<0 .
$$

Using (35), (36) and the definitions of the matrices $\Pi_{\xi \xi}$, $\Theta_{\xi \xi}$ and $\Lambda_{\xi \xi}$ given by (21), (38) and (39), and with the changes of variables $\mathcal{X}=P_{1}^{-1}, M_{i}=K_{i} \mathcal{X}, N_{i}=P_{2} L_{i}$, it is easy to obtain the inequalities given in Theorem 1 . Finally, the inequality (31) is satisfied if the optimization problem given by (12) under the LMI constraints (13) and (14) has a solution, which ends the proof.

Remark 1. After solving the optimization problem given in Theorem 1, the input-to-state stability condition given in (31) is satisfied. Thus, in the case of time varying faults with a bounded first time derivative, the state $x(t)$, the state estimation error $e_{x}(t)$ and the fault estimation error $e_{f}(t)$ converge to a ball, centered at the origin, defined by the terms $\delta$ and $\varepsilon$. The radius of the ball in which $\tilde{x}$ converges can be minimized by a choice of the parameter $\Gamma$ that minimizes $\delta$ without changing $\varepsilon$ (that does not depend on $\Gamma$ ). It thus improves the accuracy of the estimation.

Remark 2. The objective of fault tolerant control is to compensate the faults, so it is important to estimate them as soon as possible with a good accuracy. The adaptive observer studied in this paper can be considered an improvement of the classical PI observer, in the sense that the convergence of the state and fault estimates is proved (in a ball centered at the origin) even in a nonconstant fault case, whereas the assumption of a constant fault is needed to prove the convergence of the estimation error when using a PI observer (Koenig and Mammar, 2002; Ichalal et al., 2009). Note that, if the fault $f(t)$ is constant, then $f_{1 \max }=0$ and with $280=0$. Consequently, the asymptotic stability is achieved, since $\dot{V}(t)<0$ for every $\tilde{x}(t)$.

\section{Simulation example}

To illustrate the performances of the proposed approach, let us consider the system (4) defined by the matrices

$$
\begin{array}{rlrl}
A_{1} & =\left(\begin{array}{cc}
0 & 1 \\
17.2941 & 0
\end{array}\right), & A_{2}=\left(\begin{array}{cc}
0 & 1 \\
3.5361 & 0
\end{array}\right), \\
B_{1}=\left(\begin{array}{c}
0 \\
-0.1765
\end{array}\right), & B_{2}=\left(\begin{array}{c}
0 \\
-0.1763
\end{array}\right), \\
C & =I_{2} .
\end{array}
$$

The weighting functions are given by

$$
\begin{aligned}
& \mu_{1}(x(t))=1-\frac{2}{\pi}\left|x_{1}(t)\right|, \\
& \mu_{2}(x(t))=1-\mu_{1}(x(t)) .
\end{aligned}
$$


Let us consider the fault $f(t)$ defined as follows:

$$
f(t)=\left\{\begin{array}{cr}
0, & t \leq 20 \\
7.5 \sin (2 t+2.1)+15, & 20 \leq t \leq 70 \\
-0.89 u(t), & 70 \leq t \leq 100
\end{array}\right.
$$

For $t \geq 70 \mathrm{~s}$, the fault $f(t)$ describes a loss of the effectiveness of the actuator, satisfying Assumption A3. The first simulation is obtained by synthesizing a classical controller without taking the faults into account, $u(t)=$ $-\sum_{i=1}^{r} \mu_{i}(x(t)) K_{i} x(t)$, using an approach proposed by Tanaka and Wang (2001). The gains $K_{i}$ are obtained by $K_{i}=M_{i} P^{-1}$, where $P$ and $M_{i}$ are solutions of the LMIs

$$
\mathbb{S}\left(P A_{i}-B_{i} M_{j}\right)<0, \quad i, j=1,2 .
$$

With this control law, as shown in Fig. 11 the system states converge to zero in the fault-free case (i.e., for $t<20$ ), but in the faulty case the system performances are degraded from $t=20 \mathrm{~s}$ to $t=70 \mathrm{~s}$ and the system becomes unstable for $t \geq 70 \mathrm{~s}$.

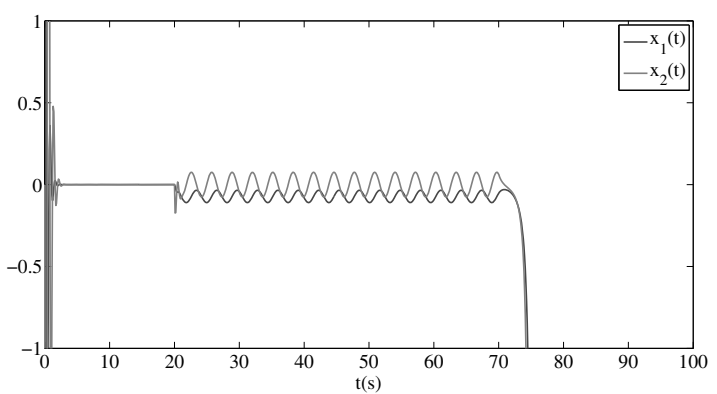

Fig. 1. System states with classical control.

The proposed fault tolerant control is designed by solving the optimization problem of Theorem 1 . For that purpose, the parameter values $\sigma=0.8, \Gamma=100$ and $\beta=10$ are chosen. The obtained gains of the observer and the controller are

$$
\begin{gathered}
L_{1}=\left(\begin{array}{cc}
0.52 & 1.22 \\
17.24 & 0.27
\end{array}\right), L_{2}=\left(\begin{array}{ll}
0.52 & 1.21 \\
3.48 & 0.26
\end{array}\right), \\
F_{1}=\left(\begin{array}{cc}
3.63 & -43.25
\end{array}\right), F_{2}=\left(\begin{array}{ll}
3.62 & -43.20
\end{array}\right), \\
K_{1}=\left(\begin{array}{ll}
-161.89 & -65.95
\end{array}\right), \\
K_{2}=\left(\begin{array}{ll}
-156.25 & -65.24
\end{array}\right) .
\end{gathered}
$$

Figure 2 illustrates the results of the proposed control law obtained after solving the optimization problem of Theorem 1. One can note that, with the fault $f(t)$ defined in (44), the performances are better than those of the classical control and the system remains stable for $t \geq 70$ (Fig. 2, top). The observer rapidly and accurately estimates the fault as shown in Fig. 2 (bottom).
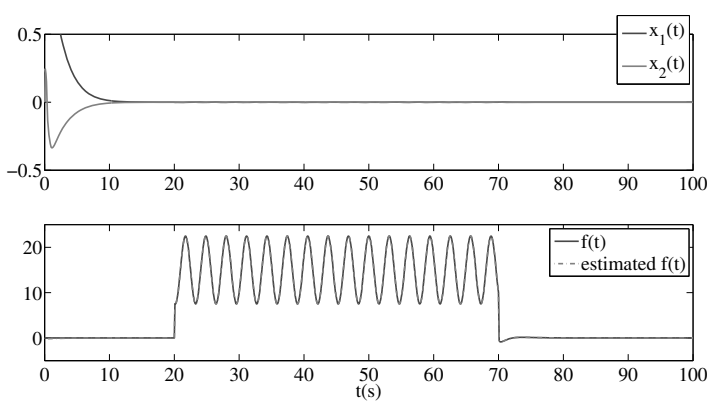

Fig. 2. Fault tolerant control: system states (top), fault and its estimate (bottom).

In this example, the classical control cannot preserve the stability of the system when $\gamma \geq 0.89$. However, based on simulations not reported here due to space limitation, it can be claimed that the proposed FTC strategy can tolerate faults until $\gamma=0.992$, which means that if the loss in the effectiveness of the actuator is less than $99.2 \%$, the proposed controller makes the system stable. In Fig. 3. after $t>70$ the loss of the effectiveness of the actuator is considered with $\gamma=0.99$, and we see that the controller compensates it despite its severity.
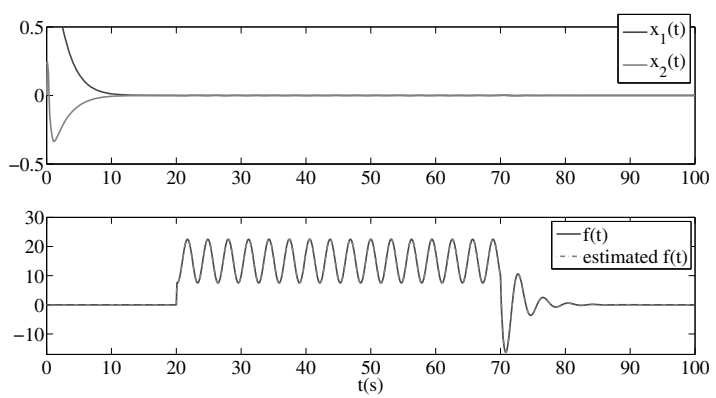

Fig. 3. Fault tolerant control with $\gamma=0.99$ : system states (top), fault and its estimate (bottom).

In addition, this approach provides a rapid and accurate estimate of the actuator faults present with the adaptive observer (Fig. 2, bottom), which constitutes an FDI block for diagnosis. If $f(t)=7.5 \sin (2 t+$ $2.1)+15$, its derivative over time is bounded by 15. Then, in this simulation example, the term $\delta=$ $1 / \sigma f_{1 \max }^{2} \lambda_{\max }\left(\Gamma^{-1} G^{-1} \Gamma^{-1}\right)=0.0186$, and the term $\varepsilon$ can be minimized by an appropriate choice of $\Gamma$ to reduce the radius of the ball in which the estimation errors converge and then obtain a more accurate fault estimate.

\section{Conservatism reduction with Polya's theorem}

In the previous section, the proposed result may be conservative in the sense that common Lyapunov matrices 
were sought to satisfy $r^{2}$ LMIs. Recently, a new interesting method to reduce the conservativeness of the matrix summations inequality has been proposed with the use of Polya's theorem (Sala and Ariño, 2007). Let us consider the inequality

$$
\Delta_{\xi \xi}=\sum_{i=1}^{r} \sum_{j=1}^{r} \mu_{i}(\xi(t)) \mu_{j}(\xi(t)) \Delta_{i j}<0
$$

where $\Delta_{i j}$ is defined in Eqn. (29).

Noticing that $\left(\sum_{i=1}^{r} \mu_{i}(\xi(t))\right)^{p}=1$ for any positive integer $p$, it can be deduced that inequalities like $\Delta_{\xi \xi}<0$ can be rewritten as $\left(\sum_{i=1}^{r} \mu_{i}(\xi(t))\right)^{p} \Delta_{\xi \xi}<0$. By gathering all terms on the left hand side of the inequality with the same coefficient, less conservative LMI conditions than $\Delta_{i j}<0$ for $i, j=1, \ldots, r$ can be obtained. As proved by Sala and Ariño (2007), if a solution exists for a given $p$, it satisfies the inequalities obtained for $p+1$. As a consequence, increasing $p$ will provide less restrictive conditions and, if $p \rightarrow+\infty$, asymptotic necessary and sufficient conditions for the negativity of (46) are obtained. The authors also proposed an algorithm to compute finite values of $p$, which gives necessary and sufficient conditions with a given accuracy. The reader can refer to the paper by Sala and Ariño (2007) for more details on a Polya theorem based relaxation approach.

Theorem 2. Under Assumption 1, given positive scalars $\sigma$ and $\beta$, if there exist symmetric and positive definite matrices $\mathcal{X} \in \mathbb{R}^{n \times n}, P_{2} \in \mathbb{R}^{n \times n}, G \in \mathbb{R}^{n_{u} \times n_{u}}$, matrices $M_{i} \in \mathbb{R}^{n_{u} \times n}$ and $N_{i} \in \mathbb{R}^{n \times n_{y}}$ and a positive scalar $\eta$ as a solution to the optimization problem

$\min \eta$

subject to

$$
\begin{gathered}
\left(\begin{array}{cc}
\eta I & B_{i}^{T} P_{2}-F_{i} C \\
* & \eta I
\end{array}\right)>0, \\
\mathcal{Q}_{i i}<0, \quad i=1, \ldots, r, \\
3 \mathcal{Q}_{i i}+\mathcal{Q}_{i j}+\mathcal{Q}_{j i}<0, \quad i, j=1, \ldots, r, \quad i \neq j, \\
3 \mathcal{Q}_{i i}+\mathcal{Q}_{j j}+3 \mathcal{Q}_{i j}+3 \mathcal{Q}_{j i}<0, \\
i, j=1, \ldots, r, \quad i \neq j, \\
6 \mathcal{Q}_{i i}+3 \mathcal{Q}_{i j}+3 \mathcal{Q}_{i k}+3 \mathcal{Q}_{j i}+3 \mathcal{Q}_{k i}+\mathcal{Q}_{j k} \\
+\mathcal{Q}_{k j}<0, \quad i, j, k=1, \ldots, r, i<j<k, \\
3 \mathcal{Q}_{i i}+3 \mathcal{Q}_{j j}+6 \mathcal{Q}_{i j}+6 \mathcal{Q}_{j i}+3 \mathcal{Q}_{i k}+3 \mathcal{Q}_{k i} \\
+3 \mathcal{Q}_{j k}+3 \mathcal{Q}_{k j}<0, \quad r, i<j<k \\
i, j, k=1, \ldots, \quad r, i<
\end{gathered}
$$

$$
\begin{aligned}
& 6 \mathcal{Q}_{i i}+6 \mathcal{Q}_{i j}+6 \mathcal{Q}_{j i}+6 \mathcal{Q}_{i k} \\
& +6 \mathcal{Q}_{k i}+6 \mathcal{Q}_{i l}+6 \mathcal{Q}_{l i}+3 \mathcal{Q}_{j k} \\
& +3 \mathcal{Q}_{k j}+3 \mathcal{Q}_{j l}+3 \mathcal{Q}_{l j}+3 \mathcal{Q}_{k l}+3 \mathcal{Q}_{l k}<0, \\
& \quad i, j, k=1, \ldots, r, \quad i<j<k<l, \\
& 6\left(\mathcal{Q}_{i j}+\mathcal{Q}_{j i}+\mathcal{Q}_{i k}+\mathcal{Q}_{k i}+\mathcal{Q}_{i l}+\mathcal{Q}_{l i}+\mathcal{Q}_{i m}\right. \\
& +\mathcal{Q}_{m i}+\mathcal{Q}_{j k}+\mathcal{Q}_{k j}+\mathcal{Q}_{j l}+\mathcal{Q}_{l j}+\mathcal{Q}_{j m} \\
& \left.+\mathcal{Q}_{m j}+\mathcal{Q}_{k l}+\mathcal{Q}_{l k}+\mathcal{Q}_{k m}+\mathcal{Q}_{m k}\right)<0 \\
& \quad i, j, k, l, m=1, \ldots, r, \quad i<j<k<l<m,
\end{aligned}
$$

where $\mathcal{Q}_{i j}$ is defined in (14), then the system $x(t)$, the state estimation error $e_{x}(t)$ and the fault estimation error $e_{f}(t)$ are bounded. The gains of the observer and the fault tolerant control are given by $F_{i}, L_{i}=P_{2}^{-1} N_{i}$ and $K_{i}=$ $M_{i} \mathcal{X}^{-1}$

Proof. According to Theorem 1, the solution of the FTC problem is obtained by minimizing $\eta$ subject to the constraints (13) and $\sum_{i=1}^{r} \sum_{j=i}^{r} \mu_{i}(\xi(t)) \mu_{j}(\xi(t)) Q_{i j}<0$, which, due to the convexity of the weighting functions, is equivalent to

$$
\left(\sum_{k=1}^{r} \mu_{k}(\xi(t))\right)^{p} \sum_{i=1}^{r} \sum_{j=i}^{r} \mu_{i}(\xi(t)) \mu_{j}(\xi(t)) \mathcal{Q}_{i j}<0 .
$$

Setting $p=3$ and gathering the terms sharing the same combinations of weighting functions, we obtain

$$
\begin{aligned}
\sum_{i=1}^{r} & \sum_{j=1}^{r} \mu_{i} \mu_{j} \mathcal{Q}_{i j}=\left(\sum_{i=1}^{r} \mu_{i}\right)^{3} \sum_{i=1}^{r} \sum_{j=1}^{r} \mu_{i} \mu_{j} \mathcal{Q}_{i j} \\
= & \sum_{i=1}^{r} \mu_{i}^{5} \mathcal{Q}_{i i}+\sum_{\substack{i, j=1 \\
i \neq j}}^{r} \mu_{i}^{4} \mu_{j}\left(3 \mathcal{Q}_{i i}+\mathcal{Q}_{i j}+\mathcal{Q}_{j i}\right) \\
& +\sum_{i, j=1}^{r} \mu_{i}^{3} \mu_{j}^{2} \tilde{Q}_{i j}+\sum_{i=1}^{r} \sum_{\substack{j=1 \\
i<j}}^{r} \sum_{\substack{k=1 \\
j<k}}^{r} \mu_{i}^{3} \mu_{j} \mu_{k} \tilde{Q}_{i j k} \\
& +\sum_{i=1}^{r} \sum_{\substack{j=1 \\
i<j}}^{r} \sum_{\substack{k=1 \\
j<k}}^{r} \mu_{i}^{2} \mu_{j}^{2} \mu_{k} \tilde{Q}_{i j k}^{*} \\
& +\sum_{i=1}^{r} \sum_{\substack{j=1 \\
i<j}}^{r} \sum_{\substack{k=1 \\
j<k}}^{r} \sum_{\substack{l=1 \\
k<l}}^{r} \mu_{i}^{2} \mu_{j} \mu_{k} \mu_{l} \tilde{Q}_{i j k l} \\
& +\sum_{i=1}^{r} \sum_{\substack{j=1 \\
i<j}}^{r} \sum_{\substack{k=1 \\
j<k}}^{r} \sum_{\substack{l=1 \\
k<l}}^{r} \sum_{\substack{m=1 \\
l<m}}^{r} \mu_{i} \mu_{j} \mu_{k} \mu_{l} \mu_{m} \tilde{Q}_{i j k l m}
\end{aligned}
$$

with

$$
\begin{aligned}
\tilde{Q}_{i j}= & 3 \mathcal{Q}_{i i}+\mathcal{Q}_{j j}+3 \mathcal{Q}_{i j}+3 \mathcal{Q}_{j i} \\
\tilde{Q}_{i j k}= & 6 \mathcal{Q}_{i i}+3\left(\mathcal{Q}_{i j}+\mathcal{Q}_{j i}+\mathcal{Q}_{i k}+\mathcal{Q}_{k i}\right) \\
& +\mathcal{Q}_{j k}+\mathcal{Q}_{k j},
\end{aligned}
$$




$$
\begin{aligned}
\tilde{Q}_{i j k}^{*}= & 3 \mathcal{Q}_{i i}+3 \mathcal{Q}_{j j}+6 \mathcal{Q}_{i j}+6 \mathcal{Q}_{j i}+3 \mathcal{Q}_{i k} \\
& +3 \mathcal{Q}_{k i}+3 \mathcal{Q}_{j k}+3 \mathcal{Q}_{k j} \\
\tilde{Q}_{i j k l}= & 6\left(\mathcal{Q}_{i i}+\mathcal{Q}_{i j}+\mathcal{Q}_{j i}+\mathcal{Q}_{i k}+\mathcal{Q}_{k i}\right. \\
& \left.+\mathcal{Q}_{i l}+\mathcal{Q}_{l i}\right) \\
\tilde{Q}_{i j k l m}= & 6\left(\mathcal{Q}_{i j}+\mathcal{Q}_{j i}+\mathcal{Q}_{i k}+\mathcal{Q}_{k i}+\mathcal{Q}_{i l}+\mathcal{Q}_{l i}\right. \\
& +\mathcal{Q}_{i m}+\mathcal{Q}_{m i}+\mathcal{Q}_{j k}+\mathcal{Q}_{k j}+\mathcal{Q}_{j l} \\
& +\mathcal{Q}_{l j}+\mathcal{Q}_{j m}+\mathcal{Q}_{m j}+\mathcal{Q}_{k l}+\mathcal{Q}_{l k} \\
& \left.+\mathcal{Q}_{k m}+\mathcal{Q}_{m k}\right) .
\end{aligned}
$$

Finally, with the same reasoning as in Theorem 1, the optimization problem with relaxed LMI constraints of Theorem 2 is obtained.

Remark 3. The use of Polya's theorem leads to increasing the number of LMIs to be solved. It is the price to pay to have less conservative stability conditions. With present powerful computers it is possible to solve a great number of LMIs in an acceptable computation time. Accordingly, for a given system, it suffices to take a minimal value of $p$ guaranteeing the existence of a solution to the LMIs. As shown in the above simulation example, a solution exists without using Polya's theorem $(p=0)$. If we are faced with an unfeasible problem, an iterative procedure can be used by increasing the integer value $p$ and, once the problem is feasible, the obtained value of the parameter $p$ is the minimal one, so it is not necessary to further increase this parameter.

\section{Fault tolerant control by trajectory tracking}

In this section the control objective is not only closed-loop stabilization, but also trajectory tracking. First, the FTC strategy is detailed, before addressing the FTC design.

7.1. FTC strategy. The state trajectory $x(t)$ to be followed by the controlled system is given by a reference model which corresponds to the model of the fault-free system with nominal input $u(t)$ defined by

$$
\left\{\begin{aligned}
\dot{x}(t) & =\sum_{i=1}^{r} \mu_{i}(\xi(t))\left(A_{i} x(t)+B_{i} u(t)\right) \\
y(t) & =\sum_{i=1}^{r} \mu_{i}(\xi(t)) C_{i} x(t) .
\end{aligned}\right.
$$

Because of the fault $f(t)$, the state of the faulty controlled system, denoted by $x_{f}(t)$, may differ from the reference trajectory. As a consequence, the control input of the system has to be modified, and the resulting applied input is denoted $u_{f}(t)$. The faulty system is given by

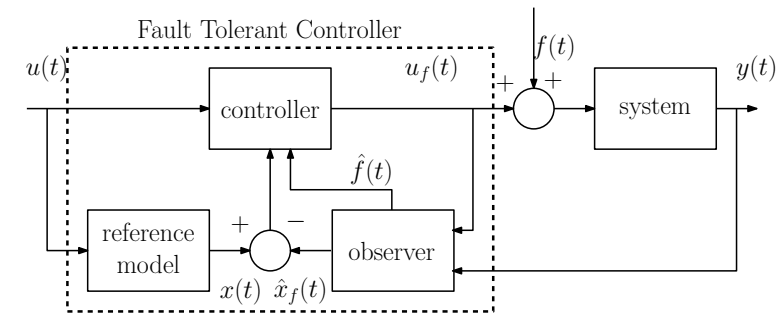

Fig. 4. Fault tolerant control scheme.

$$
\left\{\begin{array}{l}
\dot{x}_{f}(t)=\sum_{i=1}^{r} \mu_{i}\left(\xi_{f}(t)\right)\left(A_{i} x_{f}(t)+B_{i}\left(u_{f}(t)+f(t)\right)\right), \\
y_{f}(t)=\sum_{i=1}^{r} \mu_{i}\left(\xi_{f}(t)\right) C_{i} x_{f}(t) .
\end{array}\right.
$$

The faults considered are the same as in the previous sections. Note that the weighting functions depend on a faulty premise variable $\xi_{f}(t)$. The latter may be the system input, which depends on the state $x_{f}(t)$ or the output $y_{f}(t)$. Consequently, the fault necessarily affects these variables. In order to minimize the state deviation due to the fault, the controlled input $u_{f}(t)$ encompasses two additive terms depending on the fault estimate and on the estimated state deviation $x(t)-\hat{x}_{f}(t)$. One should note that the reference state is simulated from (51), and thus it is accessible for the control law, whereas the faulty system state has to be estimated. The FTC law $u_{f}(t)$ is given by

$$
u_{f}(t)=-\hat{f}(t)+K\left(x(t)-\hat{x}_{f}(t)\right)+u(t) .
$$

A PI observer is designed to provide the controller with both the estimates of the actuator fault and of the faulty system state. The proposed PI observer is given by

$$
\left\{\begin{aligned}
\dot{\hat{x}}_{f}(t)= & \sum_{i=1}^{r} \mu_{i}\left(\xi_{f}(t)\right)\left(A_{i} \hat{x}_{f}(t)+B_{i}\left(u_{f}(t)+\hat{f}(t)\right)\right. \\
& \left.+H_{1 i}\left(y_{f}(t)-\hat{y}_{f}(t)\right)\right) \\
\dot{\hat{f}}(t)= & \sum_{i=1}^{r} \mu_{i}\left(\xi_{f}(t)\right)\left(H_{2 i}\left(y_{f}(t)-\hat{y}_{f}(t)\right)\right) \\
\hat{y}_{f}(t)= & \sum_{i=1}^{r} \mu_{i}\left(\xi_{f}(t)\right) C_{i} \hat{x}_{f}(t) .
\end{aligned}\right.
$$

The premise variable $\xi_{f}(t)$ is assumed to be known and the observer weighting functions depend on the same premise variable as the system (52).

The overall scheme of the proposed FTC strategy is depicted by Fig. 4 The FTC design consists in determining the control law (53) and the PIO (54), such that the controlled system state $x_{f}(t)$ is as close to the reference state $x(t)$ as possible.

Remark 4. A fixed gain in the control law (53) has been chosen, rather than a PDC-like control law involving 
premise variables. Indeed, if the premise variable $\xi_{f}$ is the control signal $u_{f}(t)$, implementing such a control law is not possible (in that case the control signal $u_{f}(t)$ depends on weighting functions, which themselves depend on the control signal). This problem is not encountered if the premise variable is $\xi_{f}(t)=y_{f}(t)$. But the choice of the control law structure (53) unifies the case of measurable premise variables for both $u_{f}(t)$ and $y_{f}(t)$.

7.2. FTC design. The FTC design consists in determining the gains $K$ in (53) as well as $H_{1 i}$ and $H_{2 i}$ in (54) that minimize the trajectory tracking error defined by $e(t)=x(t)-x_{f}(t)$ and the state and fault estimation errors, respectively defined by $e_{x}(t)=x_{f}(t)-\hat{x}_{f}(t)$ and $e_{f}(t)=f(t)-\hat{f}(t)$. From (51)-(54) and the definition of the errors, it follows that

$$
\begin{aligned}
\dot{x}(t)= & \sum_{i=1}^{r} \mu_{i}\left(\xi_{f}(t)\right)\left(A_{i} x(t)+B_{i} u(t)\right)+\delta(t), \\
\dot{x}_{f}(t)= & \sum_{i=1}^{r} \mu_{i}\left(\xi_{f}(t)\right)\left(A_{i} x_{f}(t)+B_{i}(u(t)\right. \\
& \left.\left.+e_{f}(t)+K\left(e(t)+e_{x}(t)\right)\right)\right), \\
\dot{\hat{x}}_{f}(t)= & \sum_{i=1}^{r} \sum_{j=1}^{r} \mu_{i}\left(\xi_{f}(t)\right) \mu_{j}\left(\xi_{f}(t)\right)\left(A_{i} \hat{x}_{f}(t)\right. \\
& +B_{i}\left(u(t)+K\left(e(t)+e_{x}(t)\right)\right) \\
& \left.+H_{1 i} C_{j} e_{x}(t)\right),
\end{aligned}
$$

where $\delta(t)$ is defined by

$$
\delta(t)=\sum_{i=1}^{r}\left(\mu_{i}(\xi(t))-\mu_{i}\left(\xi_{f}(t)\right)\right)\left(A_{i} x(t)+B_{i} u(t)\right) .
$$

Assuming $\dot{f}(t)=0$, the error dynamics is given by

$$
\dot{\tilde{e}}(t)=\left(\begin{array}{cc}
A_{\xi_{f}}-B_{\xi_{f}} K & -\tilde{L}_{\xi_{f}} \\
0 & \tilde{A}_{\xi_{f}}-H_{\xi_{f}} \tilde{C}_{\xi_{f}}
\end{array}\right) \tilde{e}(t)+\tilde{\Gamma} \delta(t) .
$$

with

$$
\begin{array}{rlrl}
\tilde{e}(t) & =\left(\begin{array}{c}
e(t) \\
e_{x}(t) \\
e_{f}(t)
\end{array}\right), & \tilde{\Gamma}=\left(\begin{array}{c}
I_{n} \\
0 \\
0
\end{array}\right), \\
H_{i}=\left(\begin{array}{c}
H_{1 i} \\
H_{2 i}
\end{array}\right), & \tilde{L}_{i}=\left(\begin{array}{ll}
B_{i} K & B_{i}
\end{array}\right), \\
\tilde{A}_{i}=\left(\begin{array}{cc}
A_{i} & B_{i} \\
0 & 0
\end{array}\right), & \tilde{C}_{i}=\left(\begin{array}{ll}
C_{i} & 0
\end{array}\right) .
\end{array}
$$

In order to design the PIO, it is necessary that the pairs $\left(\tilde{C}, \tilde{A}_{i}\right)$ be observable or at least detectable.

Remark 5. One can note that, in the previous section, the weighting functions depend on the premise variable $\xi_{f}(t)$. It can be an external known variable which is not affected by faults. Indeed, Witczak et al. (2008) proposed a method for this case with an application to the three tank system in open-loop control. In this case, $\xi(t)=\xi_{f}(t)$ and Eqn. (59) becomes an autonomous system

$$
\dot{\tilde{e}}(t)=\left(\begin{array}{cc}
A_{\xi_{f}}-B_{\xi_{f}} K & -\tilde{L}_{\xi_{f}} \\
0 & \tilde{A}_{\xi_{f}}-H_{\xi_{f}} \tilde{C}_{\xi_{f}}
\end{array}\right) \tilde{e}(t) .
$$

In Takagi-Sugeno modeling, it is often considered that the premise variable $\xi(t)$ is the input, the output or the state of the system, which are necessarily affected by faults. Consequently, $\xi(t) \neq \xi_{f}(t)$ and the fault and state estimation errors as well as the state tracking error are expressed by (59). When $\xi(t)=u(t)$ and $\xi_{f}(t)=u_{f}(t)$, the term $\delta(t)$ does not converge to zero if $x_{f}(t)$ converges to the reference state $x(t)$, but if $\xi(t)=y(t)$ and $\xi_{f}(t)=y_{f}(t)$, the tolerant control allows the convergence of $x_{f}(t)$ to $x(t)$ and $y_{f}(t)$ to $y(t)$. Then the term $\delta(t)$ also converges to zero, which gives better results compared with the case where $\xi(t)=u(t)$. The same problem can appear if the output is also affected by faults. In these cases, the fault tolerant control design aims to minimize the difference between $x_{f}(t)$ and $x(t)$ and to minimize the $\mathcal{L}_{2}$ gain of the transfer from $\delta(t)$ to the state tracking error.

The gains $K, H_{1 i}$ and $H_{2 i}$ are determined by solving the optimization problem under LMI constraints given in the next theorem.

Theorem 3. Consider $\lambda$ to be a positive scalar. The system (59) that generates the state tracking error $e(t)$ and the state and fault estimation errors $e_{x}(t)$ and $e_{f}(t)$ is stable and the $\mathcal{L}_{2}$-gain of the transfer from $\delta(t)$ to $e(t)$ is bounded if there exists symmetric and positive definite matrices $X_{1}, X_{2}$ and $P_{2}$, matrices $\bar{H}_{i}$ and $\bar{K}$ as well as a positive scalar $\bar{\gamma}$ solution to the following optimization problem

$$
\min _{X_{1}, X_{2}, P_{2}, \bar{K}_{i}, \bar{H}_{i}} \bar{\gamma}
$$

subject to

$$
\begin{aligned}
& Y_{i i}<0, \quad i=1, \ldots, r, \\
& \frac{1}{r-1} Y_{i i}+Y_{i j}+Y_{j i}<0, \quad i<j,
\end{aligned}
$$

where

$$
\begin{gathered}
Y_{i j}=\left(\begin{array}{ccccc}
\Psi_{i} & -B_{i} M & 0 & I_{n} & X_{1} \\
* & -2 \lambda X & \lambda I_{n+n_{u}} & 0 & 0 \\
* & * & \Delta_{i j} & 0 & 0 \\
* & * & * & -\bar{\gamma} I_{n} & 0 \\
* & * & * & * & -I_{n}
\end{array}\right)<0 \\
\Psi_{i}=\mathbb{S}\left(A_{i} X_{1}-B_{i} \bar{K}\right), \\
\Delta_{i j}=\mathbb{S}\left(P_{2} \tilde{A}_{i}-\bar{H}_{i} \tilde{C}_{i j}\right),
\end{gathered}
$$




$$
M=\left(\begin{array}{ll}
\bar{K} & X_{2}
\end{array}\right), \quad X=\left(\begin{array}{cc}
X_{1} & 0 \\
0 & X_{2}
\end{array}\right) .
$$

The controller and observer gains are computed from

$$
H_{i}=\left(\begin{array}{c}
H_{1 i} \\
H_{2 i}
\end{array}\right)=P_{2}^{-1} \bar{H}_{i}, \quad K=\bar{K} X_{1}^{-1},
$$

and the $\mathcal{L}_{2}$-gain from $\delta(t)$ (58) to the tracking error $e(t)$ is obtained by

$$
\gamma=\sqrt{\bar{\gamma}}
$$

Proof. With the variable changes $X_{1}=P_{1}^{-1}, \bar{K}=K X_{1}$, $\bar{H}_{i}=P_{2} H_{i}, M=\left[\begin{array}{ll}\bar{K} & X_{2}\end{array}\right]$, which implies

$$
B_{i} M=\tilde{L}_{i}\left(\begin{array}{cc}
X_{1} & 0 \\
0 & X_{2}
\end{array}\right)=\tilde{L}_{i} X,
$$

and a Schur complement, the inequalities (63) are equivalent to

$$
\left(\begin{array}{ccc}
\Xi_{i} & -\tilde{L}_{i} X & I_{n} \\
* & -2 \lambda X-\lambda^{2} \Delta_{i j}^{-1} & 0 \\
* & * & -\gamma^{2} I_{n}
\end{array}\right)<0,
$$

where $\Delta_{i}$ is defined in (65) and $\Xi_{i}$ given by

$$
\Xi_{i}=\mathbb{S}\left(A_{i} P_{1}^{-1}-B_{i} K P_{1}^{-1}\right)+P_{1}^{-1} P_{1}^{-1} .
$$

If (63) hold, then the $(3,3)$ blocks of $Y_{i}$, i.e., $\Delta_{i}$, are negative definite and

$$
\begin{aligned}
& \left(X+\lambda \Delta_{i j}^{-1}\right)^{T} \Delta_{i j}\left(X+\lambda \Delta_{i j}^{-1}\right) \leq 0 \\
& \quad \Leftrightarrow X \Delta_{i j} X \leq-\lambda\left(X+X^{T}\right)-\lambda^{2} \Delta_{i j}^{-1} .
\end{aligned}
$$

Consequently, 69) implies

$$
\left(\begin{array}{ccc}
\Xi_{i} & -\tilde{L}_{i} X & I_{n} \\
* & X \Delta_{i j} X & 0 \\
* & * & -\gamma^{2} I_{n}
\end{array}\right)<0
$$

Pre- and post-multiplying (72) by $\operatorname{diag}\left(P_{1}, X^{-1}, I_{n}\right)$, it follows that 63 implies

$$
\mathcal{N}_{i j}=\left(\begin{array}{ccc}
\mathbb{S}\left(\Lambda_{i}\right)+I_{n} & -P_{1} \tilde{L}_{i} & P_{1} \\
* & \Delta_{i j} & 0 \\
* & * & -\gamma^{2} I_{n}
\end{array}\right)<0
$$

with $\Lambda_{i}$ defined by $\Lambda_{i}=P_{1} A_{i}-P_{1} B_{i} K$. Choosing a quadratic Lyapunov function defined by

$$
V(\tilde{e}(t))=\tilde{e}^{T}(t)\left(\begin{array}{cc}
P_{1} & 0 \\
0 & P_{2}
\end{array}\right) \tilde{e}(t)
$$

with $P_{1} \in \mathbb{R}^{n \times n}$ and $P_{2} \in \mathbb{R}^{\left(n+n_{u}\right) \times\left(n+n_{u}\right)}$ real symmetric positive definite matrices, it is easily derived from (59) that

$$
\begin{aligned}
\dot{V}(\tilde{e}(t))+e^{T}(t) e(t) & -\gamma^{2} \delta^{T}(t) \delta(t) \\
& =\left(\begin{array}{c}
e(t) \\
\delta(t)
\end{array}\right)^{T} \mathcal{N}_{i j}\left(\begin{array}{c}
e(t) \\
\delta(t)
\end{array}\right) .
\end{aligned}
$$

As a consequence, (63) implies

$$
\dot{V}(\tilde{e}(t))+e^{T}(t) e(t)-\gamma^{2} \delta^{T}(t) \delta(t)<0,
$$

which is well known to be a sufficient condition for the $\mathcal{L}_{2}$-gain from $\delta(t)$ to $e(t)$ to be bounded by $\gamma$ and for $\tilde{e}(t)$ to asymptotically converge toward zero when $\delta(t)$ is identically null. Finally, the application of Tuan's lemma (Tuan et al., 2001), and minimization of $\gamma$ leads to the the optimization problem with LMI constraints given in Theorem 3 .

Remark 6. The conservatism of the results given in the previous theorem could be reduced by applying, in a similar way, Polya's theorem presented in the first strategy.

Remark 7. The assumption that the fault signal is constant over time is restrictive, but in many practical situations where faults are slowly time-varying signals, the estimation of the faults is correct and the proposed FTC scheme can be applied. In the case where faults are not slowly time-varying or constant, the PIO can be replaced by a Proportional Multiple Integral Observer (PMIO). Such an observer was introduced in order to filter highfrequency disturbance by Ibrir (2004). It is able to estimate a large class of time-varying signals, which satisfies the assumption $f^{(q+1)}(t)=0$. The principle of this observer is based on the estimation of the first $q$ derivatives of the signal $f(t)$. This observer can also be extended to the case where $f^{(q+1)}(t)$ is bounded (see Ichalal et al., 2009). It is also possible to use the adaptive observer given in the first part of the paper, since, as shown in the example, this observer can estimate faults with fast variations.

\section{Simulation examples}

In this section, two examples are proposed to illustrate the proposed FTC. The first discusses some aspects of the proposed approach and the second is dedicated to the actuator fault tolerant control of lateral dynamics of a vehicle.

Example 1. To illustrate the proposed actuator fault tolerant control strategy for T-S systems with measurable premise variables and affected by actuator faults, two academic examples are presented.

First case: $\xi(t)=u(t)$. Consider the T-S system described by

$$
\left\{\begin{array}{l}
\dot{x}_{f}(t)=\sum_{i=1}^{r} \mu_{i}(u(t))\left(A_{i} x_{f}(t)+B_{i}\left(u_{f}(t)+f(t)\right)\right), \\
y_{f}(t)=C x_{f}(t)+\omega(t)
\end{array}\right.
$$

where

$$
A_{1}=\left[\begin{array}{ccc}
-2 & 1 & 1 \\
1 & -3 & 0 \\
2 & 1 & -8
\end{array}\right], A_{2}=\left[\begin{array}{ccc}
-3 & 2 & -2 \\
0 & -3 & 0 \\
5 & 2 & -4
\end{array}\right] \text {, }
$$




$$
B_{1}=\left[\begin{array}{l}
0 \\
1 \\
1
\end{array}\right], \quad B_{2}=\left[\begin{array}{l}
1 \\
1 \\
0
\end{array}\right], C=\left[\begin{array}{lll}
1 & 1 & 1 \\
1 & 0 & 1
\end{array}\right] .
$$

The weighting functions depend on the input $u(t)$, which is the nominal control of the system in the fault-free case. They are defined by $\mu_{1}(u(t))=(1-u(t)) / 2$ and $\mu_{2}(u(t))=1-\mu_{1}(u(t))$. To apply the proposed FTC strategy, the following reference model is considered:

$$
\left\{\begin{array}{l}
\dot{x}(t)=\sum_{i=1}^{r} \mu_{i}(u(t))\left(A_{i} x(t)+B_{i} u(t)\right), \\
y(t)=C x(t) .
\end{array}\right.
$$

The fault $f(t)$ is time varying and defined as follows:

$$
f(t)=\left\{\begin{array}{lr}
0, & t<10 \\
-0.5 u(t), & 10 \leq t \leq 20, \\
1, & 20<t
\end{array}\right.
$$

and $\omega(t)$ is a random signal whose maximum value corresponds to $10 \%$ of the maximum amplitude of the measured signal $y(t)$. Notice that even if the assumption $\dot{f}(t)=0$ is not satisfied, the PIO is able to reconstruct time varying signals with slow variation.

Solving the optimization problem under LMI constraints in Theorem 3 with $\lambda=20$ results in the following matrices:

$$
\begin{aligned}
& H_{11}=\left[\begin{array}{cc}
-24.84 & 59.47 \\
30.05 & -29.75 \\
31.54 & -43.02
\end{array}\right], \\
& H_{12}=\left[\begin{array}{cc}
-11.03 & 45.34 \\
31.58 & -33.25 \\
17.80 & -26.25
\end{array}\right] \text {, } \\
& H_{21}=\left[\begin{array}{ll}
337.82 & -356.67
\end{array}\right] \text {, } \\
& H_{21}=\left[\begin{array}{ll}
338.57 & -353.93
\end{array}\right] \text {, } \\
& K=\left[\begin{array}{lll}
6.5179 & 4.9204 & 1.2659
\end{array}\right] \text {, } \\
& \gamma=0.4721 \text {. }
\end{aligned}
$$

Figure 5 (top) shows the time evolution of the fault $f(t)$ and its estimate $\hat{f}(t)$, while the bottom part depicts the nominal control $u(t)$ and the FTC $u_{f}(t)$. The state estimation errors, $x_{f}(t)-\hat{x}_{f}(t)$, are shown in the top of Fig. 6, while the bottom part shows the state tracking errors $x(t)-x_{f}(t)$. Finally, Fig. 7 allows the comparison of the reference model states with the state obtained when the system is faulty without any modification of the control law and those of the system when using FTC.

Even if a fault occurs, the system trajectory follows that of the reference model, which represents the trajectory of the system in the fault-free situation. Thus, the FTC control law compensates the fault and allows normal functioning of the system in the presence of faults.
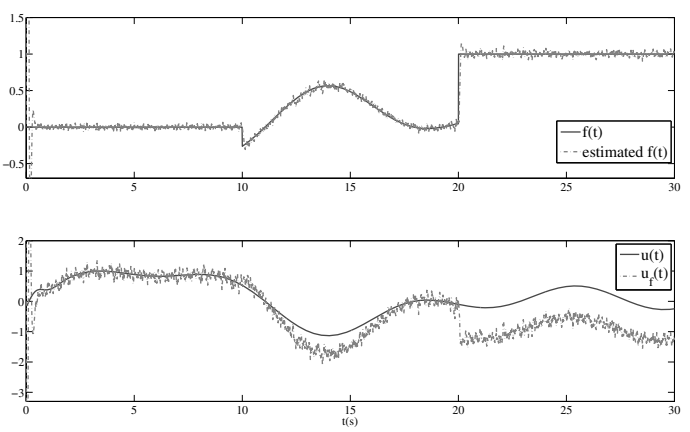

Fig. 5. Fault and its estimates (top), nominal control and FTC (bottom).
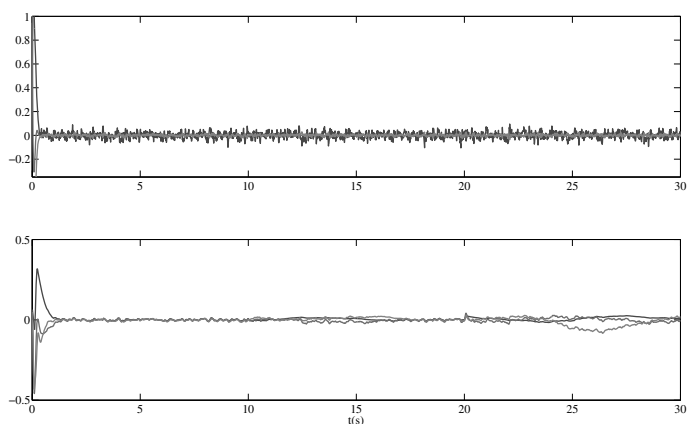

Fig. 6. State estimation errors (top), state tracking errors (bottom).

Second case: $\xi(t)=y(t)$. In this subsection, the previous system is considered, but with weighting functions depending on the first component of the system output vector. Figure 8 illustrates the state estimation errors (top) and the state tracking errors (bottom). It is clear that the use of weighting functions depending on the output of the system provides better results than the case where they depend on the control input. This is because the system is only affected by actuator faults and the perturbation like term $\delta(t)$ converges to zero when $y_{f}(t)$ converges to the reference $y(t)$. But in the previous simulation, the term $\delta(t)$ did not converge to zero, in the presence of a fault, because $u(t) \neq u_{f}(t)$, which leads to $\mu_{i}(u(t)) \neq \mu_{i}\left(u_{f}(t)\right)$. As a conclusion, considering the problem of the fault tolerant control of T-S systems with actuator faults, it is more interesting to use the output of the system as a premise variable. However, when actuator and sensor faults occur simultaneously, better results are obtained by using the system state as a premise variable. This is a more difficult and general case, but the obtained state tracking error is less than those obtained above. First results on this point were published by Ichalal et al. (2010).

Example 2. In this second example, an interesting model is used. Security and assistance in vehicles are important, 

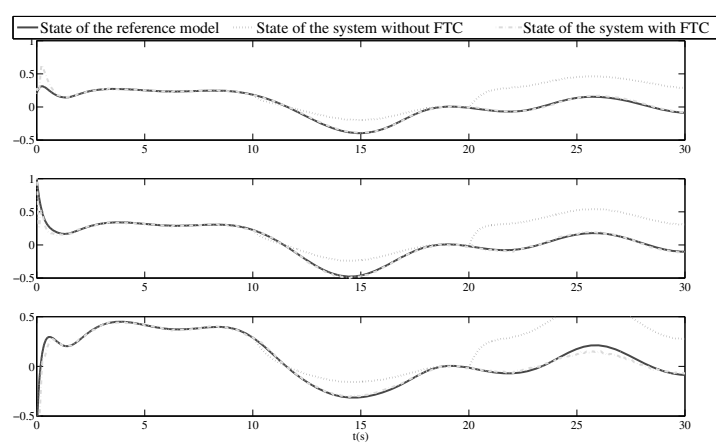

Fig. 7. Comparison between states of the system without a fault, states with a fault and nominal control and states with a fault and FTC.
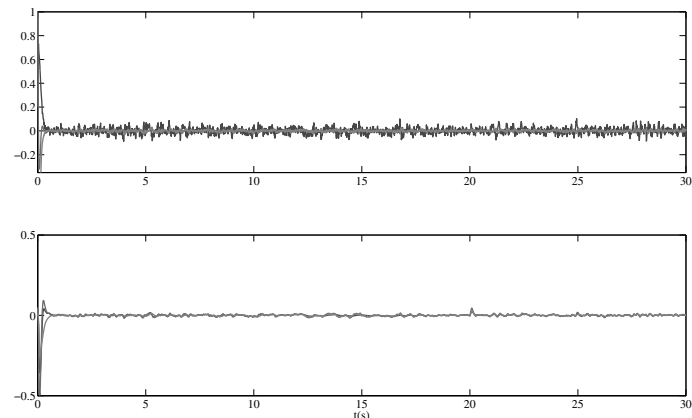

Fig. 8. State estimation errors (top), state tracking errors (bottom).

especially in dangerous situations like cornering and land keeping with high speed. For that purpose, this example deals with the problem of fault tolerant control of lateral dynamics of a vehicle in a cornering situation (for example). Let us consider the nonlinear model of the vehicle lateral dynamics given by the following differential equations:

$$
\left\{\begin{array}{l}
m \dot{v}(t)+u(t) r(t))=2\left(F_{f}(t)+F_{r}(t)\right) \\
J \dot{r}(t)=2\left(\alpha_{f} F_{f}(t)-a_{r} F r(t)\right.
\end{array}\right.
$$

where $u$ and $v$ are the longitudinal and lateral velocities $(v=\beta u), r$ is the yaw rate, $\beta$ denotes the side slip angle, $m$ and $J$ are the mass and the yaw moment of inertia, respectively, $a_{f}$ and $a_{r}$ are the distances of the front and rear axle from the center of gravity. $F_{f}$ and $F_{r}$ are front and rear lateral forces. A T-S simplified model, as given by Oudghiri et al. (2008), is represented by

$$
\left\{\begin{array}{l}
\dot{x}(t)=\sum_{i=1}^{2} \mu_{i}\left(\left|\alpha_{f}\right|\right)\left(A_{i} x(t)+B_{i} \delta_{f}\right), \\
y(t)=\sum_{i=1}^{2} \mu_{i}\left(\left|\alpha_{f}\right|\right)\left(C_{i} x(t)+D_{i} \delta_{f}\right),
\end{array}\right.
$$

where $x^{T}=\left[\begin{array}{ll}v & r\end{array}\right], \delta_{f}$ is the steering angle of the front wheel.
The output $y$ contains the lateral acceleration and yaw rate given by the inertial unit and expressed as functions of state variables. The matrices defining the model are

$$
\begin{array}{ll}
A_{1}=\left(\begin{array}{cc}
-6.9426 & -0.8775 \\
26.5175 & -7.8343
\end{array}\right), & B_{1}=\left(\begin{array}{c}
3.4892 \\
39.2914
\end{array}\right), \\
A_{2}=\left(\begin{array}{cc}
-0.4735 & -0.9971 \\
0.6356 & -0.4921
\end{array}\right), & B_{2}=\left(\begin{array}{c}
0.2767 \\
3.1155
\end{array}\right), \\
C_{1}=\left(\begin{array}{cc}
-6.9426 & 2.4491 \\
0 & 1
\end{array}\right), & D_{1}=\left(\begin{array}{c}
69.7847 \\
0
\end{array}\right), \\
C_{2}=\left(\begin{array}{cc}
-0.4735 & 0.0587 \\
0 & 1
\end{array}\right), & D_{2}=\left(\begin{array}{c}
5.5333 \\
0
\end{array}\right) .
\end{array}
$$

The weighting functions depend on the slip angle $\alpha_{f}$, which is assumed to be known. An additive actuator fault signal affecting the vehicle is given in the form

$$
f(t)=\left\{\begin{array}{lr}
0, & t<10 \\
-0.1 \sin (0.314 t), & 10 \leq t<14.95 \\
0.1, & t \geq 14.95
\end{array}\right.
$$

Furthermore, a random noise with a maximal magnitude 0.05 is added to output measurements. Figure 9 illustrates a comparison between the states of the reference model (without a fault), the states of the faulty vehicle without FTC, and finally the states of the vehicle with FTC. Clearly, the proposed strategy is robust with respect to the actuator additive fault $f(t)$. In Fig. 10, we can observe the estimation of the fault. Moreover, the FTC scheme provides good results in the presence of measurement noise, as shown in the figures. Figure 11 illustrates the state estimation of vehicle states.

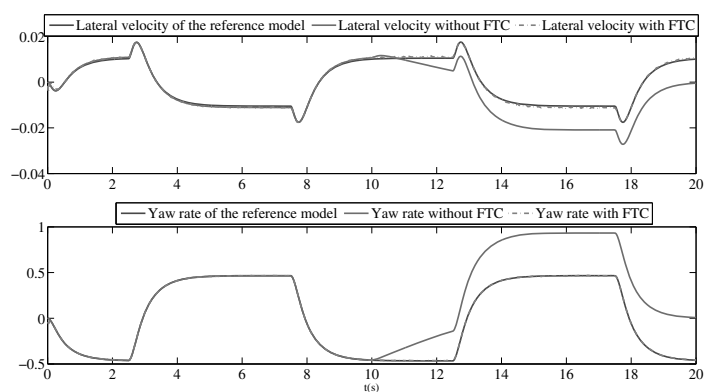

Fig. 9. States of the reference model, vehicle model without FTC and with FTC.

\section{Conclusions and future work}

This paper was dedicated to the study of a new actuator fault tolerant control for nonlinear systems described by a Takagi-Sugeno model. Stabilizing active fault tolerant control was studied. It requires simultaneous estimations of the state and fault, obtained by the proposed adaptive 

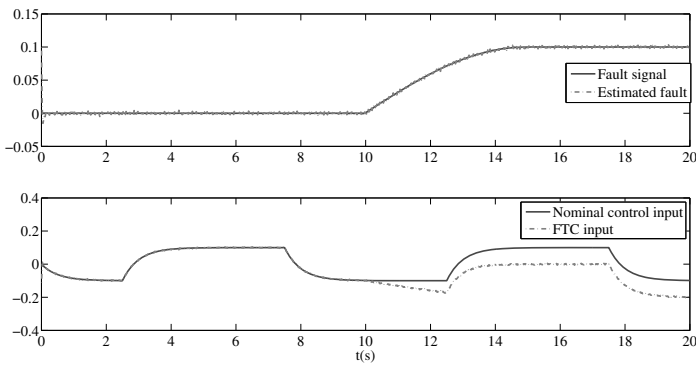

Fig. 10. Fault estimation and control inputs.
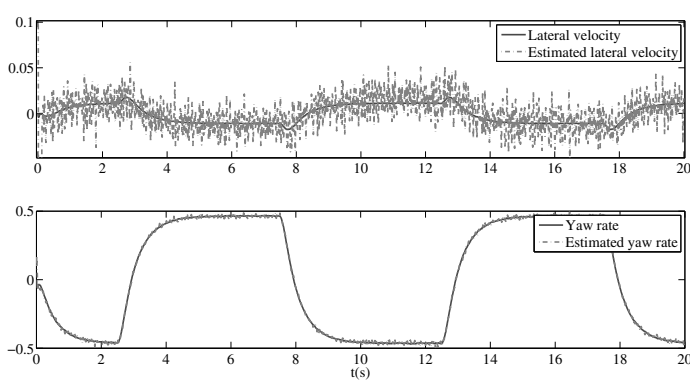

Fig. 11. State estimation.

observer. This observer is able to simultaneously estimate time varying faults and state variables with a good accuracy. Furthermore, it rapidly produces estimates which is important to preserve the performances of the system. The stability analysis, made with Lyapunov theory and input-to-state stability, was performed in the case of time varying faults, and, furthermore, asymptotic stability was guaranteed in the case of constant faults. Sufficient stability conditions were given in terms of LMIs. In order to reduce the conservatism of the given conditions, Polya's theorem was used to derive relaxed conditions for the FTC design for nonlinear systems.

Secondly, an extension of the first work was proposed in order to provide a new control law which forces the faulty-state of the system to track a reference trajectory given by the fault free model of the system. For that purpose, the control law was modified in order to take into account the trajectory tracking error and the estimated actuator faults. Finally, an application to a nonlinear model of lateral dynamics of a vehicle with an additive actuator fault was given in order to illustrate the second approach.

Future works will concern FTC of systems affected by both sensor and actuator faults and/or uncertainties and/or perturbations. Secondly, the case when the premise variables are unmeasured (like states of the system) will also be investigated. It will also be interesting to study the case when a set of actuators is completely out of order. In this situation the dimensions of the matrices $B_{i}$ and of the control vector $u(t)$ are reduced.

\section{References}

Akhenak, A., Chadli, M., Ragot, J. and Maquin, D. (2008). Fault detection and isolation using sliding mode observer for uncertain Takagi-Sugeno fuzzy model, 16th Mediterranean Conference on Control and Automation, Ajaccio, France, pp. 286-291.

Corless, M. and Tu, J. (1998). State and input estimation for a class of uncertain systems, Automatica 34(6): 757-764.

Darouach, M., Zasadzinski, M. and Xu, S. (1994). Full-order observers for linear systems with unknown inputs, IEEE Transactions on Automatic Control 39(3): 606-609.

Gao, Z. and Ding, S. X. (2007). Actuator fault robust estimation and fault-tolerant control for a class of nonlinear descriptor systems, Automatica 43(5): 912-920.

Ibrir, S. (2004). Robust state estimation with q-integral observers, American Control Conference, Boston, MA, USA, pp. 3466-3471.

Ichalal, D., Marx, B., Ragot, J. and Maquin, D. (2009a). Simultaneous state and unknown inputs estimation with PI and PMI observers for Takagi-Sugeno model with unmeasurable premise variables, 17th Mediterranean Conference on Control and Automation, MED'09, Thessaloniki, Greece, pp. 353-358.

Ichalal, D., Marx, B., Ragot, J. and Maquin, D. (2010). Fault tolerant control for Takagi-Sugeno systems with unmeasurable premise variables by trajectory tracking, IEEE International Symposium on Industrial Electronics, Bari, Italy, pp. 2097-2102.

Koenig, D. and Mammar, S. (2002). Design of a proportional integral observer for unknown input descriptor systems, IEEE Transactions on Automatic Control 47(12): 20572063.

Kruszewski, A., Wang, R. and Guerra, T.M. (2008). Nonquadratic stabilization conditions for a class of uncertain nonlinear discrete time T-S fuzzy models: A new approach, IEEE Transactions on Automatic Control 53(2): 606-611.

Leith, D.J. and Leithead, W.E. (1999). Survey of gainscheduling analysis design, International Journal of Control 73(11): 1001-1025.

Luenberger, D. (1971). An introduction to observers, IEEE Transactions on Automatic Control 16(6): 596-602.

Marx, B., Koenig, D. and Ragot, J. (2007). Design of observers for Takagi-Sugeno descriptor systems with unknown inputs and application to fault diagnosis, IET Control Theory and Application 1(5): 1487-1495.

Mufeed, M.M., Jiang, J. and Zhang, Z. (2003). Active Fault Tolerant Control Systems: Stochastic Analysis and Synthesis, Springer-Verlag, Berlin/Heidelberg.

Nagy, A., Mourot, G., Marx, B., Schutz, G. and Ragot, J. (2009). Model structure simplification of a biological reactor, 15th IFAC Symposium on System Identification, SYSID'09, Saint Malo, France, pp. 257-262.

Niemann, H. and Stoustrup, J. (2005). Passive fault tolerant control of a double inverted pendulum-A case study, Control Engineering Practice 13(8): 1047-1059. 
Ocampo-Martinez, C., De Dona, J. and Seron, M. (2010). Actuator fault-tolerant control based on set separation, International Journal of Adaptive Control and Signal Processing 24(12): 1070-1090.

Oudghiri, M., Chadli, M. and El Hajjaji, A. (2008). Robust observer-based fault tolerant control for vehicle lateral dynamics, International Journal of Vehicle Design 48(34): $173-189$.

Patton, R. (1997). Fault-tolerant control systems: The 1997 situation, 3rd IFAC Symposium on Fault Detection, Supervision and Safety of Technical Processes, Hull, UK, pp. 1033-1054.

Patton, R.J. and Klinkhieo, S. (2009a). An LPV approach to active FTC of a two-link manipulator, 7th Workshop on Advanced Control and Diagnosis, Zielona Góra, Poland.

Patton, R. and Klinkhieo, S. (2009b). Actuator fault estimation and compensation based on an augmented state observer approach, 48th IEEE Conference Decision and Control/28th Chinese Control Conference, CDC/CCC 2009, Shanghai, China, pp. 8482-8487.

Sala, A. and Ariño, C. (2007). Asymptotically necessary and sufficient conditions for stability and performance in fuzzy control: Applications of Polya's theorem, Fuzzy Sets and Systems 158(24): 2671-2686.

Stilwell, D.J. and Rugh, W.J. (1997). Interpolation of observer state feedback controllers for gain scheduling, IEEE Transactions on Automatic Control 44(6): 1225-1229.

Takagi, T. and Sugeno, M. (1985). Fuzzy identification of systems and its applications to modeling and control, IEEE Transactions on Systems, Man, and Cybernetics 15(1): 116-132.

Tanaka, K. and Wang, H. (2001). Fuzzy Control Systems Design and Analysis: A Linear Matrix Inequality Approach, John Wiley and Sons, Hoboken, NJ.

Tuan, H., Apkarian, P., Narikiyo, T. and Yamamoto, Y. (2001). Parameterized linear matrix inequality techniques in fuzzy control system design, IEEE Transactions on Fuzzy Systems 9(2): 324-332.

Witczak, M., Dziekan, L., Puig, V. and Korbicz, J. (2008). Design of a fault-tolerant control scheme for Takagi-Sugeno fuzzy systems, 16th Mediterranean Conference on Control and Automation, Ajaccio, France, pp. 280-285.

Zhang, K., Jiang, B. and Cocquempot, V. (2008). Adaptive observer-based fast fault estimation, International Journal of Control, Automation, and Systems 6(3): 320-326.

Zhang, K., Jiang, B. and Shi, P. (2009). A new approach to observer-based fault-tolerant controller design for TakagiSugeno fuzzy systems with state delay, Circuits, Systems, and Signal Processing 28(5): 679-697.

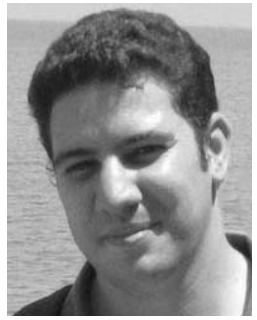

Dalil Ichalal received his Master's degree from Paul Cézanne Aix Marseille 3 University (France) in 2006, and a Ph.D. degree from the National Polytechnic Institute of Lorraine (France) in 2009. In 2010, he joined the Department of Electrical Engineering of the University of Evry Val d'Essonne (France) and the IBISC (Informatique, Biologie Integrative et systèmes complexes) Laboratory. His research interests include state estimation and observer design, as well as fault diagnosis and fault tolerant control of nonlinear systems with application to vehicles and motorcycles.

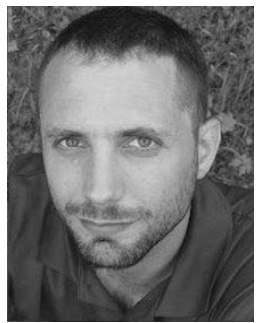

Benoît Marx was born in 1977, obtained an engineering degree and an M.Sc. degree, both in automatic control, in 2000 from Ecole Centrale de Nantes (France), and the Ph.D. degree from the Grenoble Institute of Technology (France) in 2003. Since September 2004 he has been an assistant professor of automatic control at the University of Lorraine (France) and a member of the Research Centre for Automatic Control of Nancy. His research interests include state estimation, fault diagnosis and fault tolerant control of singular and/or nonlinear systems, with particular attention paid to nonlinear systems represented by multiple-models.

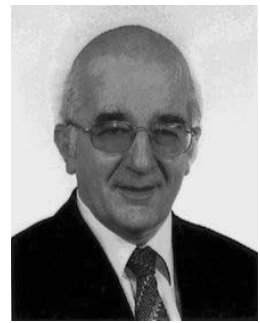

José Ragot is a researcher in the Research Center for Automatic Control of Nancy. His major research fields include data validation and reconciliation, process diagnosis, fault detection and isolation. A part of his activities is devoted to modeling and state estimation adapted to process diagnosis and mainly in the field of multi-models. $\mathrm{He}$ has successfully advised $63 \mathrm{Ph} . \mathrm{D}$. and $3 \mathrm{HDR}$ (French D.Sc.) theses and published about 500 refereed technical communications, including 94 papers in international journals, 280 communications in international conferences, 4 books and 12 chapters in collective volumes. Applications have been in various fields such as mineral and metallurgical processing, chemical engineering, water treatment, aerospace, environmental processes.

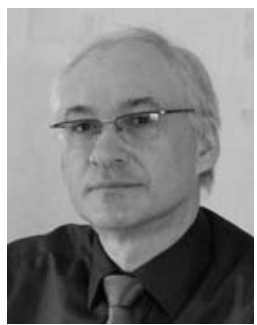

Didier Maquin is a professor of automatic control at the University of Lorraine, France. He teaches automatic control and applied mathematics in various engineering schools. $\mathrm{He}$ is a member of the Research Center for Automatic Control of Nancy and the scientific leader of one of the five research groups of a lab for dependability and system diagnosis, which gathers around twenty teachers/researchers. On the national level, Didier Maquin is involved in the French Automatic Control Research Group. He has been on its managemen staff since 2001 and currently serves as the head of the theme Dependability, Supervision and Maintenance. Didier Maquin has co-authored around 50 journal articles and 120 conference communications.

Received: 17 January 2011

Revised: 15 June 2011 